\title{
L'arbitrage Et La Compétence Exclusive De La Juridiction Etatique (Etude comparé)
}

\author{
Adnan Salih Al-omar \\ Professeur du droit commercial, Facult é de droit, Université de Yarmouk, Jordan \\ adnan.alomar@yu.edu.jo
}

Date de réception: 2/11/2020 Date de revision: 27/11/2020 Date d'acceptation: 25/12/2020 DOI: https://doi.org/DOI:10.31559/LCJS2020.1.3.5

Résumé : Lorsqu'une juridiction a obtenu de la loi compétence exclusive en certaines matières, aucune autre juridiction ne peut se prononcer sur les questions qui relèvent de cette compétence que la question ait été portée devant cette juridiction en tant que demande principale ou à titre de moyen de défense. La juridiction saisie doit donc surseoir à statuer jusqu'à ce que la juridiction ayant la compétence exclusive ait statué.

Également, la compétence judiciaire exclusive est un critère souvent invoqué dans les droits étatiques pour constater l'arbitrabilité des litiges. L'arbitrage est seulement prohibé si la matière porte sur le contentieux ayant un caractère objectif et si les intérêts du tiers sont en jeu. Donc il est exclu dès qu'une matière ressort exclusivement de la compétence d'une juridiction de l’Etat. Aussi, la compétence judiciaire pour connaître d'une matière déterminée est considérée comme étant d'ordre public, donc inarbitrable.

Mots-clés : l'arbitrabilité ; inarbitrable ; compétence ; étatiques.

\section{Introduction}

Lorsqu'une juridiction a obtenu de la loi compétence exclusive en certaines matières, aucune autre juridiction ne peut se prononcer sur les questions qui relèvent de cette compétence que la question ait été portée devant cette juridiction en tant que demande principale ou à titre de moyen de défense. La juridiction saisie doit donc surseoir à statuer jusqu'à ce que la juridiction ayant la compétence exclusive ait statué1.

Également, la compétence judiciaire exclusive est un critère souvent invoqué dans les droits étatiques pour constater l'arbitrabilité des litiges. L'arbitrage est seulement prohibé si la matière porte sur le contentieux ayant un caractère objectif et si les intérêts du tiers sont en jeu ${ }^{2}$. Donc il est exclu dès qu'une matière ressort exclusivement de la compétence d'une juridiction de l'Etat. Aussi, la compétence judiciaire pour connaître d'une matière déterminée est considérée comme étant d'ordre public, donc inarbitrable.

Le recours à l'arbitrage ne serait impossible que dans le cas d'une attribution impérative de compétence ${ }^{3}$. Dans certaines législations ${ }^{4}$, les règles relatives à la reconnaissance et à l'exécution des sentences étrangères n'autorisent pas le juge à reconnaître ou à exécuter la sentence étrangère rendue en violation d'une règle de compétence impérative.

Après avoir démontré le domaine de l'exclusivité de la compétence étatique (§I), il nous conviendra de l'apprécier (§II).

\section{Paragraphe I. Domaine D’application De L'exclusivité}

L'exercice de la volonté des parties sur le partage des compétences entre les juridictions étatiques et les arbitres connaît une limite. Les mesures préparant l'exécution de la sentence à intervenir relèvent de la compétence exclusive des juridictions étatiques même lorsqu'elles interviennent à titre conservatoire. Ainsi, les parties, qui pourraient convenir qu'elles éviteront de saisir les juridictions étatiques de telles mesures avant

\footnotetext{
${ }^{1}$ H. SINAY, La compétence exclusive, JCP. 1958 I 1451.

2 J-B RACINE, L'arbitrage commercial international et l'ordre public, LGDJ, 1999. p. 91.

3 J. ROBERT, op. cit., $\mathrm{n}^{\circ} 40$.

${ }^{4}$ Article 198-1 CPC égyptien ; article 119 D.S. omanais n 13/97.
} 
l'issue du litige, ne pourraient en revanche pas confier aux arbitres le pouvoir d'ordonner une saisie ou toute autre mesure relevant de l'exécution de la décision, en raison du monopole des juridictions étatiques à ce sujet.

En fait, les matières confiées à la connaissance exclusive des juridictions étatiques sont nombreuses. En revanche, on ne pourrait pas dans cet étude les citer toutes, mais certains exemples révélateurs méritent d'être soulignes ; le premier est puisé dans le droit pénal(I), alors que le second, dans le droit privé (II).

\section{Dans Le Droit Penal}

A ce propos, le droit pénal est le meilleur exemple, puisqu'un arbitre n'a pas le pouvoir de prononcer une sanction pénale ${ }^{5}$. En fait, l'exclusivité de la compétence étatique de la justice pénale s'accorde mal avec l'idée d'une soumission à un juge privé d'un litige de cet ordré ${ }^{6}$. Néanmoins, la difficulté se pose dans le cas où la règle « le criminel tient le civil en l'état » est en cause. Cette règle s'applique à l'arbitre interne. Cependant, il est plus difficile de l'appliquer en droit international7.

La question qui pourrait être soulevée à cet égard est celle de savoir si l'arbitre international a l'obligation de se retarder à juger dans l'attente de la décision à intervenir sur l'action publique ${ }^{8}$. Le droit français positif n'impose pas une telle obligation à l'arbitre et il laisse l'arbitre apprécier librement l'opportunité de retarder sa propre décision puisque la règle « le criminel tient le civil en l'état», Cet solution, dégagé par la cour d'appel de Paris depuis quelques années ${ }^{9}$ viennent d'être consacrées sans ambiguïté par la cour de cassation dans un arrêt du 25 octobre $2005^{10}$. Il était jugé que l'article 4, alinéa 2, du Code de procédure pénale était sans application pour l'arbitre statuant en matière internationale, en raison de l'autonomie de la procédure arbitrale qui obéit à des règles propres ... dont il lui appartient d'apprécier l'opportunité11. La Cour d'appel de Paris avait seulement exclu l'application de la règle lorsque le juge pénal compétent était un juge étranger ${ }^{12}$.

En revanche, dans le cas au recours en annulation d'une sentence arbitrale, la règle « le criminel tient le civil en l'état " s'impose aux juridictions étatiques chargées du contrôle de la régularité des sentences arbitrales, dès lors que la procédure pénale se déroule en France « l'article 4 du Code de procédure pénale est applicable, même en matière internationale, au recours en annulation d'une sentence arbitrale si la procédure pénale se déroule en France ».13.

Le droit jordanien a bien consacré la règle « le criminel tient le civil en l'état»,

\section{II.- DANS LE DROIT PRIVE}

Bien des matières sont également octroyées, dans le droit privé, à la compétence exclusive des juridictions étatiques, tel que les litiges relatifs au droit de concurrence, les litiges relatifs aux brevets, les contrats maritimes, contrats de représentations commerciales, ... etc.

Pour étudier ces questions, nous considérerons des exemples, qui méritent d'être soulignés, dans un premier point dans le droit français (1), et dans le droit jordanien dans un second point (2).

\section{A.- En Droit Français}

En effet, les matières les plus connues confiées exclusivement au droit étatique sont dans le droit français : les litiges relatifs au droit de la concurrence et le droit de la propriété intellectuelle. Du point de vue des individus en présence, le droit de la concurrence et le droit de la propriété intellectuelle reposent tous deux sur un fondement mixte. D'une part, l'équité joue un rôle essentiel pour la propriété intellectuelle plus que pour la propriété ordinaire (qualité d'auteur ; droit moral). D'autre part, la propriété intellectuelle et le droit de la concurrence découlent de politiques publiques bien définies ${ }^{14}$.

\footnotetext{
${ }^{5}$ F. Nammour, Droit Et Pratique De L'arbitrage Interne Et International, 2e Edition (Refonte Complète) Lgdj, Bruylant, Delta, 2005 , P. 139.

${ }^{6}$ J. Beguin, G. Bourdeaux, A. Couret, B. Le Bass, D. Mainguy, M. Menjucq, H. Ruiz Fabri, C. Seraglini, J.M. Sorel, Op. Cit., P. 915.

${ }^{7}$ L. Lévy, A. V. Schlaepfer, La suspension d'instance dans l'arbitrage international, Cah. arb., Gaz. Pal. №318, pp. 18 -26.

${ }^{8} \mathrm{~L}$ 'action publique est l'action conduite au nom de la société en vue de réprimer une infraction en application de la loi pénale. Elle est engagée au nom de la société puisqu'elle vise à réprimer un trouble à l'ordre public et non à réparer un préjudice personnel. Elle est mise en œuvre par le ministère public, contre l'auteur ou les complices d'une infraction: Pour de plus voir site internet, http://fr.wikipedia.org/wiki/Action_publique.

9 Paris, 1re ch. C, 1er mars 2001, Rev. arb. 2001.583, République du Congo c/ SA Commisimpex, note Racine ; 17 janv. 2002 , SA Omenex c/ Hugon, Rev. arb. 2002.391, note Racine ; 20 juin 2002, Sté Ordatech c/ Sté W. Management, inédit.

10 ; Cass., 1ère civ., 25 oct. 2005, SA Omenex c. Hugon, Juris-Data n² 2005-030437, D. 2005, p. 3052 et 3060, obs. Th. Clay ; D. 2006 , p. 199, avis J. Sainte-Rose ; Rev. arb. 2006, p. 103, note J.-B. Racine; F.-X. Train, Clunet 2006, p. 937.

11 V. Eric Loquin, Le principe « le criminel tient le civil en l'état » n'est pas applicable en matière d' arbitrage international, RTD Com. 2003 p. 63 ; CA Paris, 17 janv. 2002, Rev. arb. 2002.391, note Racine ; voir enfin Cass. 1ére civ., 6 mai 2003, pourvoi nº0 00 -16.822 : Juris-Data ${ }^{\circ} 2003-$ 018901 ; JCP G 2003, IV, 2144 ; Cass., 1ère civ., 4 juin 2008, pourvoi n06-15.320.

12 Paris 30 mars 1995, RTD Com. 2003 1997.230 ; 24 janv. 1991, Rev. arb. 1992.158, obs. Cohen

13 Cass. Civ. Arrêt n 1389 du 25 octobre 2005, lixinter.net, jurisprudence 2005 à 2010 ,

14 Y. DERAINS, L'expérience de la Cour d'arbitrage de la Chambre de commerce internationale en matière de propriété industrielle, : Rev. arb., 1977 n $^{\circ} 1$ pp. $40-60$.
} 


\section{Les litiges relatifs au droit de concurrence.}

Le droit de la concurrence est une matière relevant de l'ordre public économique en ce qu'il établit des contraintes en vue de protéger le libre jeu du marché. La question de l'arbitrabilité des litiges relatifs au droit de la concurrence est complexe et controversée ${ }^{15}$. Pendant très longtemps, il a été enseigné qu'il n'était pas concevable que les arbitres s'approchent d'un problème d'entente ou d'un problème de position dominante ou d'un autre problème de droit de la concurrence. Les mentalités ont évolué, la Cour Suprême des Etats unis a ouvert la route dans une célèbre affaire Mitsubishi ${ }^{16}$ en admettant l'arbitrage en matière de droit de la concurrence.

Au plan international, il utile de rappeler que l'arrêt Mitsubishi a été le premier arrêt à reconnaître en termes généraux l'arbitrabilité des litiges en matière de droit de la concurrence ${ }^{17}$. Il a servi de détonateur et de modèle, tant pour d'autres pays de la Common Law qu'en Europe occidentale ${ }^{18}$.La Cour de Cassation française a admis l'arbitrabilité des litiges même lorsque des questions de droit de la concurrence étaient soulevées.

La question est notamment de savoir si l'arbitre a aptitude pour sanctionner le non-respect du droit de la concurrence ou si ses dispositions d'ordre public économique ressortissent à la compétence exclusive des juridictions étatiques. Quelques certitudes émergent cependant de la matière.

Tout d'abord, sous peine de voir l'arbitrage systématiquement paralysé par une partie de mauvaise foi, la simple démonstration de l'opposition ou de la contradiction du contrat avec le droit de la concurrence n'empêche pas l'arbitre de statuer. Ensuite, il est certain qu'un arbitre ne peut s'affranchir du respect des règles d'ordre public applicables à la cause et notamment donc, de celles relatives au droit de la concurrence.

La jurisprudence affirme nettement par les décisions de la Cour d'appel de Paris dans l'affaire Almira Films en 1989, dans l'affaire Ganz en 1991, et dans les arrêts Labinal19 et Aplix en 199320, que l'arbitre peut appliquer un texte de droit de la concurrence, en tirant le cas échéant les conséquences quant à la validité du contrat 21 .

Dans l'affaire Labinal, le tribunal de commerce de Paris en premier temps a rejeté l'exception d'incompétence en arguant que « les règles de la concurrence sont d'ordre public économique tant au plan communautaire que de celui de chacun des Etats membres». Toutefois, la Cour d'appel de Paris, dans un arrêt du 19 mai 1993, a reformé le jugement en énonçant le principe selon lequel : "l'arbitrabilité d'un litige n'est pas exclue du seul fait qu'une réglementation d'ordre public est applicable au rapport de droit litigieux». Et les conseillers d'en conclure que les arbitres avaient le pouvoir de " tirer les conséquences civiles d'un comportement jugé illicite au regard de règles d'ordre public pouvant être directement appliquées aux relations des parties en cause... ».

La Cour d'appel a donc reconnu dans l'arrêt Labinal le pouvoir de l'arbitre d'apprécier la validité d'un contrat au regard des règles communautaires de la concurrence de l'annuler s'il viole lesdites règles, ainsi que d'allouer des dommages et intérêts. Cette position a été reconfirmée par la cour d'appel de Paris dans différents arrêts subséquents ${ }^{22}$. La même solution a été retenue par l'arrêt Aplix en 1993, la Cour d'appel a réaffirmé les principes de l'arrêt Labinal en précisant que les arbitres ne peuvent faire application que des règles de droit communautaire bénéficiant de l'effet direct.

Il peut même, depuis le Règlement communié Européenne no 1/2003 du Conseil du 16 décembre 2002, prononcer une exemption individuelle en vertu de l'article $81 \S 3$ du Traité CE. Ce pouvoir étant désormais attribué au juge national, il est logique de le conférer également aux arbitres ${ }^{23}$. Il est dorénavant admis que dans les affaires internationales les arbitres ont la qualité d'appliquer les règles d'ordre public ainsi que d'en sanctionner la méconnaissance éventuelle. En revanche cette qualité subit des restrictions.

Cette solution est compréhensible dès lors que la compétence de l'arbitre est limitée aux litiges portant sur des droits subjectifs des parties. Ne peuvent donc être appliquées par un tribunal arbitral que les règles communautaires dont les parties peuvent directement se prévaloir ${ }^{24}$. Dans la même mesure et sous les mêmes réserves, l'arbitrabilité des litiges en matière de droit de la concurrence est également reconnue en Italie ${ }^{25}$, en Angleterre ${ }^{26}$, en Allemagne ${ }^{27}$.

15 J-CI Com. Fasc. 197, ARBITRAGE COMMERCIAL, Cote : 03,2006.

16 United States Supreme Court, 2 juillet 1985, 105 S. Ct. 3346(1985): Yearbook Commercial Arbitration, 1986, p.555.

17 Max Van Der Stoel, The role of the OSCE high commissioner on national minorities in the field on conflict prevention, Martinus Nijhoff Publishers, 2003, p125.

18 V. arrêt rendu en suisse 1975, Chambre des recours du canton de Vaud, Ampaglas/Sofia, 28 octobre 1975, Journal des tribunaux, 1881, III, p.71; aussi l'arrêt 1992 : Rev. arb., 1993, p.124. v. également ATF, XSA et Y SA c. Z SA, 13 novembre 1998, Bulletin ASA, 1999, p.529, consid. la commenté par P.Y. Tschanz et J. M. Vuillemin, « Chronique de jurisprudence étrangère. Suisse »: Rev. arb., 2002, pp. 885, 894.

${ }^{19}$ CA Paris, 19 mai 1993 : Rev. arb., 1993, p. 645, note CH. JARROSSON.

${ }^{20}$ CA Paris, 14 octobre 1993, Aplix c. Velcro: Rev. arb., 1994, p. 164, note CH. JARROSSON.

21 Max Van Der Stoel, The role of the OSCE high commissioner on national minorities in the field on conflict prevention, Martinus Nijhoff Publishers, 2003, p126.

${ }^{22}$ V. également, Paris, 30 mars 1995, Fabre et autres C. Espitalier et autre : Rev. Arb., 1996, p.131. observe. J. PELLERIN ; Paris, 6 juillet 1995, Société Pigadis c. Société prodine, D. a ff., n². 12 Octobre 1995.

${ }^{23}$ W. ABDELGAWAD, L'arbitrage international et le nouveau règlement d'application des articles 81 et 82 CE : Rev. arb., 2004, p. 253

24 J-M. JACQUET ET PH. DELEBECQUE, Droit du commerce international, Dalloz, Cours, édition 3e, 2002 . p. 390.

${ }^{25}$ Cass. 21 aout 1996, n7733. International Handbook on Commercial Arbitration. V “Italy" p.13.

26 International Handbook on commercial Arbitration, v "England" p.21. 
Par contre, l'arbitre ne peut cependant prononcer des injonctions ou des amendes ${ }^{28}$. De tels privilèges relèvent de la compétence exclusive d'autorités spécialisées (la Commission européenne pour le droit communautaire de la concurrence). Aujourd'hui, les solutions étant acquises, le contentieux sur cette question s'est tari. Les problèmes portent actuellement sur l'intensité du contrôle des sentences au regard du droit de la concurrence. Si la Cour de justice des Communautés européennes semble exigeante ${ }^{29}$, la jurisprudence française conçoit le contrôle de manière excessivement restrictive.

En effet, dans l'arrêt Thalès ${ }^{30}$ la Cour ayant décidé de ne pas annuler une sentence pour demande nouvelle, la violation du droit communautaire de la concurrence n'ayant pas été soulevée devant les arbitres. Cette jurisprudence ne se prononçait donc pas clairement. Toutefois, la Cour de cassation vient de donner une interprétation beaucoup plus éclairante de cette jurisprudence communautaire ${ }^{31}$. Un contrat d'approvisionnement est conclu en 1991 entre la société française SNF et la société néerlandaise Cytec industries BV. Elles concluent un nouveau contrat deux ans plus tard, qui contient une clause compromissoire. SNF décide de dénoncer le contrat et Cytec met en œuvre un arbitrage à Bruxelles. Par deux sentences, le tribunal arbitral déclare nul le second contrat en application de l'article $81 \mathrm{du}$ Traité CE (interdiction des pratiques contraires au libre jeu de la concurrence), déclare les parties coresponsables et condamne SNF à indemniser Cytec. Les sentences obtiennent l'exequatur et SNF fait appel des ordonnances d'exequatur. Ayant été débouté en appel, SNF a formé un pourvoi.

En effet, la Cour de cassation tranche enfin la question de l'étendue du contrôle du juge de l'annulation en précisant que pour examiner la compatibilité d'une sentence avec des règles d'ordre public, le contrôle du juge doit se limiter au « caractère flagrant, effectif et concret de la violation alléguée ». Le juge ne doit donc pas procéder à une révision au fond de la sentence et peut, à bon droit, se limiter à un contrôle des sentences au regard de l'application du droit communautaire à l'affaire soumise aux arbitres. La solution se refuse à mettre en cause l'arbitrabilité des questions d'ordre public ${ }^{32}$.

\section{Les litiges relatifs au droit de la propriété intellectuelle.}

La question d'arbitrabilité des litiges en matière de propriété intellectuelle est une question délicate ${ }^{33}$. En effet, jusqu'en 1968 il n'était pas question d'arbitrage dans le domaine de la propriété industrielle, car les affaires qui mettaient en cause des brevets étaient considérées comme des causes communicables, au sens de l'article 1004 de l'ancien Code de Procédure civile, qui ne pouvait pas faire l'objet d'arbitrage. Donc il était hors de question d'entrer en arbitrage pour des questions de brevets d'invention.

Cette impossibilité d'arbitrage a été confirmée par la loi du 31 décembre 1964 pour les marques, puis la loi du 2 janvier 1968 pour les brevets d'invention. Ces lois avaient déposé une règle de compétence exclusive des Tribunaux de Grande Instance pour les problèmes de propriété industrielle, de brevet et de marque respectivement ${ }^{34}$. Cette impossibilité restait ferme jusqu'à la réforme de l'article 2060 du Code civil, par la loi du 5 juillet 1972, qui a voulu développer les possibilités d'arbitrage, en posant la règle de l'inarbitrabilité des " matières qui intéressent l'ordre public ». Ainsi, selon la loi du 13 juillet 1978 sur les brevets d'invention, les règles de compétence exclusive «ne font pas obstacle au recours à l'arbitrage dans les conditions prévues aux articles 2059 et 2060 du Code civil »35 Pareillement, la même disposition figure dans la loi du 4 janvier 1991 pour les marques.

On pourrait dire que l'arbitrage, par ces textes, n'est pas exclu du seul fait que les litiges ressortissent normalement de la compétence exclusive des tribunaux de grande instance. Le problème reste cependant délicat parce l'effet de l'article 2060 du Code civil - qui prohibe l'arbitrage dans les matières qui intéressent l'ordre public - reste toujours pendante. Il faut donc faire le tri et regarder, dans les questions qui peuvent être soumises à l'arbitrage, ce qui est de nature à intéresser l'ordre public et ce qui ne l'est pas.

27 Ibid., v « Germany », p.9.

${ }^{28} \mathrm{~J}-\mathrm{Cl}$, Com. Domaine de validité de la convention d'arbitrage en général : l'arbitrabilité des litiges $>1^{\circ} \mathrm{L}$ 'arbitrabilité en raison de la matière litigieuse. Cote 1-2009.

${ }^{29}$ CJCE, 1 er juin 1999, aff. C-126/97, Eco Swiss: Rev. arb. 1999, p. 631, note L. Idot.

30 CA Paris, 18 nov. 2004, Thalès : Rev. arb., 2005, p. 751; JDI 2005, p. 357, note A. MOURRE, n 8, obs. CH. SERAGLINI ; Rev. crit. DIP 2006, p. 104, note S. BOLLEE.

31 Cass. 1re civ., 4 juin 2008, SNF : Rev. arb., 2008, p. 346, note I. Fadlallah ; JDI 2008, p. 1107, note A. Mourre. - Cependant en matière interne, Cass. com., 17 janv. 2006, n 03-12.382, Rev. Lamy dr. conc. avr.-juin 2006, n 7, p. 48, obs. C. NOURISSAT.

32 E. LOQUIN, Le contrôle du seul « caractère flagrant, effectif et concret » de la violation de l'ordre public international par la sentence, RTD Com. 2008 p. 521

33 M. JOSSELIN-GALL, Arbitrage et propriétés intellectuelles, Droit et Patrimoine, n¹05, juin 2002, pp. 63-72.

${ }^{34}$ Certes la doctrine s'est penchée sur le berceau de ces lois et a fait remarquer qu'il ne fallait pas a ssimiler compétence exclusive et exclusion de l'arbitrage. Mais la pratique a été extrêmement réticente, influencée qu'elle était par les anciens textes et n'a pas cherché à innover, v. l'arbitrage et propriété intellectuelle, Conférence donnée le 2février 1994 devant le Groupe Rhône Alpes de Propriété Industrielle (GRAPI) par Me Pierre VÉRON, avocat ( $L A M Y$, VÉRON, RIBEYRE \& Associés), ancien Président du GRAPI, Vice-Président du entre d'Arbitrage Rhône Alpes (CAM) : http://www.veron.com/files/publications/Arbitrage_et_propriete_intellectuelle_pdf_texte.pdf.

35 Article L615-17 du Code de la propriété intellectuelle, Version consolidée au 6 août 2008. 
En dépit de ces caractéristiques, l'arbitrabilité de principe des litiges de propriété industrielle a été admise ${ }^{36}$. De manière générale, la cour d'appel de Paris a décidé que "les litiges portant sur les contrats relatifs à l'exploitation des brevets, qu'il s'agisse d'interprétation ou d'exécution du contrat, sont arbitrables" ${ }^{\prime \prime}$. Une telle solution est naturellement transposable au contentieux de l'exploitation d'une marque. De même, les litiges relatifs à la propriété d'un brevet ou d'une marque ainsi que ceux portant sur les intérêts civils d'une contrefaçon sont arbitrables ${ }^{38}$.

Toutefois, une question pourrait revêtir une importance particulière dans ce domaine, les arbitres ont-ils le pouvoir de déclarer qu'une marque ou qu'un brevet est nul ?

En matière des brevets et des marques, la solution classique consiste à refuser aux arbitres compétence pour se prononcer sur la validité du titre : "le brevet est un acte administratif délivré par l'autorité publique, il est inconcevable que des personnes privées - fussent-elles investies de la confiance des parties à un litige - tranchent sur la validité d'un titre délivré par l'autorité publique » ${ }^{39}$, le contentieux de la validité du titre était classiquement inarbitrable ${ }^{40}$. Leur compétence s'étendant seulement aux relations contractuelles, y compris celles qui découleraient de l'annulation prononcée par un juge. Les solutions ont aujourd'hui évolué. La cour d'appel de Paris a en effet autorisé l'arbitre à se prononcer sur la validité d'un brevet à titre incident ${ }^{41}$. Les juges parisiens ont déclaré :

« La question de la validité du brevet débattue de manière incidente à l'occasion d'un litige de nature contractuelle peut, ainsi que la relève l'arbitre, lui être soumise, l'invalidité éventuellement constatée n'ayant, pas plus que s'il

s'agissait de la décision d'un juge, d'autorité de la chose jugée, car elle ne figure notamment pas au dispositif, qu'elle n'a d'effet qu'à l'égard des parties, de même d'ailleurs qu'une décision en faveur de la validité, les tiers pouvant toujours demander la nullité du brevet pour les mêmes causes ». C'est une solution essentielle qui clarifie

le droit français sur cette question. Il ne semble pas en revanche que l'arbitre soit autorisé à statuer à titre principal sur la validité d'un brevet.

En cas de procédure collective organisant la faillite, si elle est ouverte en France, seule la juridiction étatique est compétente. Selon la cour de cassation, ${ }^{42}$ le principe de suspension individuelle des poursuites est d'ordre public interne et international ${ }^{43}$.

\section{B. En Droit Jordanien}

L'arbitrabilité des litiges relative à des contrats de représentations ou d'agence commerciale, contrats maritimes et contrats des distributions reçoivent des solutions défavorables, a fortiori d'hostilité caractérisée, aussi bien en Jordanie que dans plusieurs pays arabes ${ }^{44}$. Les exemples méritent d'être soulignés à cet égard : tout d'abord, les matières dans les contrats de représentations(a), ensuite dans contrats des commerces maritimes $(b)$.

\section{La représentation commerciale}

En effet, avant la loi de contrats d'agence et d'intermédiations commerciales de 2001, le décret-loi no 44 de 1985 portantes réglementations des activités d'agence commerciale avec les mandants étrangers régissait les activités de ces agents dans leurs seuls rapports avec les seules sociétés étrangères. L'article 20 de cette loi disposait : "nonobstant toute clause contraire, les juridictions du lieu d'exercice des activités de l'agent sont compétentes pour connaître de tout litige qui découle d'un contrat de représentation commercial».

Toutefois, cet article a été interprétait étroitement par la jurisprudence jordanienne. La Cour de cassation jordanienne jugeait dans l'affaire $n 411 / 8445$ au sujet d'un arbitrage qui se déroulait en Suède et qui opposait un agent commercial jordanien à une société suédoise, que la compétence des juridictions jordaniennes est d'ordre public, donc impossible d'être écartée par une clause d'arbitrage.

Il est très utile de rappeler que cette solution était aussi confirmée par d'autres arrêts de la même cour: par un arrêt de 1991 la Cour cassation ${ }^{46}$ se fondait sur l'article-(2) de la Convention de New York pour soumettre

\footnotetext{
${ }^{36}$ V. G. BONET ET CH. JARROSSON, L'arbitrabilité des litiges de propriété industrielle en droit français, in Arbitrage et propriété intellectuelle, Colloque de l'IRPI : Librairies techniques, 1994, p. 61.

${ }^{37}$ CA Paris, 24 mars 1994, Deko : Rev. arb., 1994, p. 515, note CH. JARROSSON.

${ }^{38} \mathrm{~J}-\mathrm{Cl}$, Convention d'arbitrage, Domaine de validité de la convention d'arbitrage en général : l'arbitrabilité des litiges $>1^{\circ} \mathrm{L}^{\prime}$ arbitrabilité en raison de la matière litigieuse. Cote 1-2009.

${ }^{39}$ L'arbitrage et propriété intellectuelle, Conférence donnée le 2février 1994 devant le Groupe Rhône Alpes de Propriété Industrielle (GRAPI) par Me Pierre VÉRON, avocat (LAMY, VÉRON, RIBEYRE \& Associés), ancien Président du GRAPI, Vice-Président du Centre d'Arbitrage Rhône Alpes (CAM). : http://www.veron.com/files/publications/Arbitrage_et_propriete_intellectuelle_pdf_texte.pdf.

40 CA Paris, 3 févr. 1992 : RTD com. 1993. p. 293, obs. E. LOQUIN.

${ }^{41}$ CA Paris, 28 févr. 2008, Liv Hidravlika : JurisData nº 2008-359055 ; D. 2008, p. 1325, note R. MEESE.

42 Cass. 1 re Civ., 5 févr. 1991 : Rev. arb., 1991.625, note, L. IDOT.

43 J-M. JACQUET et PH. DELEBECQUE, Droit du commerce international, Dalloz, Cours, édition 3e, 2002. pp. 390 \& 391.

${ }^{44} \mathrm{~N}$. NAJJAR, L'arbitrage dans les pays arabes face aux exigences du commerce international, L.G.D.J, 2004, p.164.

${ }^{45} \mathrm{http}: / /$ www.lob.gov.jo/ui/principles/search_no.jsp?no=411\&year=1984\&PrincType=7\&PrincPage $=000152$.

${ }^{46}$ http://www.lob.gov.jo/ui/principles/search_no.jsp?no=47\&year=1991\&PrincType $=7 \&$ PrincPage $=001856$.
} 
l'arbitrabilité du litige a la loi du lieu de l'exécution de la sentence arbitrable en ces termes : « la politique générale du Royaume hachémite jordanien en matière de représentation commerciale est la protection du citoyen et l'affirmation de la souveraineté nationale. En vertu de l'article 20 de la loi sur la représentation commerciale, le Tribunal de première instance d'Amman est le tribunal compétent pour trancher le litige portant sur les indemnités résultant du fait de la résiliation d'un contrat de représentation commerciale, lorsque la représentation exerce ses activités dans le Royaume et plus particulièrement dans la ville d'Amman »

En revanche, la nouvelle loi no 28 de 2001 de contrats d'agence et d'intermédiations commerciales, abroge le décret-loi no 44 de 1985, et dispose en son article $16:$ : a- Les juridictions jordaniennes sont compétentes pour reconnaître de tout litige ou différend résultant d'un contrat d'agence commerciale ou en application de la présente de cette loi.»

Il est vrai que l'expression "nonobstant toute clause contraire» de l'ancien article 20 ait été supprimée. Toutefois, la compétence d'attribution des tribunaux jordaniens n'a pas été changée par l'article 16 de la nouvelle loi. Ce nouvel arrangement de la loi ne décrète pas de compétence exclusive des juridictions jordaniennes pour connaitre des litiges relatifs aux contrats d'agence commerciale; en revanche, il est très susceptible, vue l'attribution spécifique de la compétence ratione materiae aux tribunaux jordaniens, que celle-ci soit considérée d'ordre public, donc exclusive de toute autre. « La jurisprudence antérieure risque d'être reconduite sous l'empire de la nouvelle loi $»^{47}$.

Néanmoins, il faudrait préciser que le droit jordanien connaissait certains caractères sous l'empire de l'ancienne loi sur l'arbitrage de 1953 toujours valables. La jurisprudence jordanienne quant à elle considérait qu'étant conclu postérieurement à la naissance du litige, le compromis d'arbitrage relatif à un contrat d'agence commercial demeurait valable. En vertu du compromis, les parties renoncent à un droit acquis dont elles ont la libre disposition ${ }^{48}$. Les parties pouvaient de même recourir à l'arbitrage, lorsque le différend portant sur un contrat d'agence commercial échappait à la loi no 44 de 1985, à condition toutefois que cet arbitrage se déroule en Jordanie sous la protection des juridictions jordaniennes.

La Cour de cassation dans son arrêt n $92 / 92^{49}$ a confirmé une jurisprudence constante selon laquelle les parties sont libres de recourir à l'arbitrage interne pour résoudre les litiges relatifs à l'exécution du contrat d'agence commercial, bien que la compétence de principe revienne aux juridictions étatiques.

Plus récemment la Cour de cassation dans son arrêt no 2486 de 199950, a confirmé également que la soumission d'un litige entre un agent commercial et une société étrangère a l'arbitrage en exécution d'une clause compromissoire, doit être interprétée comme un renoncement de sa part a la compétence des juridictions jordaniennes, même si la clause compromissoire stipule que les lois applicables sont celles du Yémen. Il n'existe pas d'interdiction a l'application de la loi d'un Etat, a condition toutefois que se conforme aux conditions prescrites par le Code civil jordanien ${ }^{51}$.

Ces contrats de droit interne étaient régis par la loi no 23 de 1975 relative aux agents et distributeurs commerciaux. En effet, les deux solutions seront sans doute reconduites sous l'empire des deux nouvelles lois, aussi bien celle qui se rapporte a l'arbitrage que la loi no 28 de 2001 précitée ${ }^{52}$.

\section{Les contrats des commerces maritimes}

De même, le droit maritime fait l'objet d'une compétence judiciaire impérative faisant obstacle a l'arbitrage, tel que le droit maritime en Jordanie et plusieurs pays d'Arabes.

La Cour de cassation jordanienne a décidé que « ... la clause compromissoire insérée dans un contrat d'affrètement maritime en vertu de laquelle les parties conviennent de soumettre le litige à un tribunal arbitral à l'étranger-est nul en vertu de l'article 215 du code de commerce maritime, qui prohibe toute clause confiant la résolution d'un litige découlant dudit contrat à une juridiction étrangère. Cette clause viole une règle de compétence exclusive des juridictions jordaniennes pour tout litige relatif au transport maritime et aux contrats d'affrètement maritime $»^{53}$. Donc on pourrait dire que la solution est bien affirmée en droit jordanien par la jurisprudence ${ }^{54}$.

La Cour de cassation syrienne également a affirmé que « la clause compromissoire insérée dans une charte en partie nulle, de nullité absolue, parce qu'elle a pour but d'évincer la compétence exclusive des juridictions

${ }^{47}$ N. NAJJAR, L'arbitrage dans les pays arabes, op.cit., p.169.

48 V. S. SALEH, commercial agency \& Distributorship in the Arab Middle East, op.cit., fasc. 4, p.24.

${ }^{49}$ Cass. Civil Jord. ${ }^{\circ} 92 / 92$, du 14 février 1993, RLAAI, n ${ }^{\circ}$ p.79.

${ }^{50}$ http://www.lob.gov.jo/ui/principles/search_no.jsp?year=1999\&no=2486\&PrincType $=7 \&$ PrincPage $=000348 \&$ publish_year=2002.

51 G. HAZBOUN, For legal consultations, Middle East Newsletter, n ${ }^{\circ}$. September. 2001.

52 N. NAJJAR, L'arbitrage dans les pays arabes face aux exigences du commerce international, L.G.D.J, 2004, p.169.

$53 \mathrm{http}$ //www.lob.gov.jo/ui/principles/search_no.jsp?no=619\&year=1990\&PrincType=7\&PrincPage $=002106$.

${ }^{54}$ http://www.lob.gov.jo/ui/principles/search_no.jsp?year=1985\&no=496\&PrincType $=7 \&$ PrincPage=001919\&publich_year=1987. 
syriennes consacrées a l'article 212 du code maritime syrien ${ }^{55}$, cette solution a été aussi consacrée par la jurisprudence en droit syrien ${ }^{56}$.

\section{Paragraphe II.- L'appreciation De L'exclusivite De La Competence Etatique}

Il n'est pas sans intérêt de révéler à cet égard que les juges étatiques, praticiens et auteurs peuvent contester l'arbitrabilité du litige en vertu de deux arguments essentiels. Si le premier est fondé sur les matières relevantes de l'ordre public (I), le second, fondées sur la prohibition des conventions (II).

\section{Les Matieres Relevant De L'ordre Public}

En effet, cet argument est fondé sur le critère des "matières insusceptibles de transaction », ou sur la prohibition dans "les matières qui intéressent l'ordre public »57. Qualifiées des lois de police et de sûreté, touchant à l'ordre public, les matières qui font l'objet de cette réglementation sont retirées du domaine de l'arbitrage. La violation d'une règle à caractère d'ordre public provoque donc l'inarbitrabilité du litige.

Il est normal que l'Etat soit fort pour protéger certains domaines d'activité nationaux en décrétant des lois de police et de sûreté. En revanche, il n'est pas contesté qu'une disposition visant à garantir un ressortissant contre toute clause en vertu de laquelle il renoncerait à un régime légal de protection ne suffit pas pour éliminer l'arbitrage international en la matière ${ }^{58}$.

L'argument découlant également de la fonction de l'arbitrage international, est un argument milite aussi contre l'adéquation du critère fondé sur les « matières qui intéressent l'ordre public » ${ }^{59}$, celui-ci n'est qu'un mode de résolution des conflits, une justice privée qui conduit aussi bien à l'application d'une loi étrangère au fond du litige qu'a la désignation de la loi de police et de sûreté du juge requis.

Il faudrait rappeler qu'il est donc inexact de considérer l'arbitrage, soi-même, comme un abandon au bénéfice de l'application du droit étatique. Sans compter que le juge dispose toujours, au stade post-arbitral, du pouvoir de sanctionner une sentence rendue en méconnaissance des exigences de son ordre public. En effet, les statistiques de la CCI confirment ces affirmations : en matière de représentation commerciale, la très grande majorité des sentences rendues par les arbitres internationaux applique au fond du litige, à défaut de choix des parties, la loi du lieu d'exécution du contrat ${ }^{60}$.

Dans une sentence CCI no $8606^{61}$, l'arbitre a repoussé l'argument d'une des parties qui contestait l'arbitrabilité d'un litige relatif a un contrat de représentation commerciale exclusive avec une partie libanaise, au motif que : "Le caractère 'nationaliste' de l'article 5 du D.-L. n 34/67 dénoncé par certains est généralement justifié par le fait que le recours aux tribunaux étatiques en toutes circonstances, garantit l'application du droit libanais et notamment du D.-L. n 34/67». " D'ailleurs, il semble bien que c'est là une des justifications d'un arrêt de la Cour d'appel de Beyrouth, en date du 30 juin 1994, qui est en retrait par rapport à l'arrêt de la Cour de cassation précitée. La Cour d'appel semble avoir fait preuve d'une certaine réticence à l'admission des clauses compromissoires au principal motif que l'arbitre étranger pourrait être amené à faire application d'un autre système juridique que le droit libanais. En l'espèce, le souci du législateur demeure totalement préservé par l'application du droit libanais au présent litige ».

En effet, l'arbitre a admis l'arbitrabilité du litige, à tel point que sa décision s'inscrivait dans le prolongement des préoccupations du législateur libanais, en l'occurrence l'application de la loi libanaise au fond du litige ${ }^{62}$. Cette solution n'est pas isolée. Dans toutes les affaires dénombrées impliquant un contrat de représentation commercial avec une partie libanaise, la loi retenue par les arbitres au fond du litige a été la loi du lieu d'exécution ${ }^{63}$.

\section{La Prohibition Des Conventions}

Il n'est sans intérêt à cet égard de relever qu'il n'est pas plus convaincant les parties, en vertu d'une convention d'arbitrage, renoncent ou modifient une compétence judiciaire exclusive, même s'il n'est pas controversé depuis les années 1950 que le critère d'exclusive n'est pas pertinent ${ }^{64}$ le caractère impératif de la compétence des tribunaux étatiques n'affecte pas l'arbitrage ; celui-ci ne saurait être assimilé à une compétence

\footnotetext{
55 Cass. civ. syrienne., n³66/1905, du 12 décembre 1980, Al-Mouhamoun 1981. 305.

56 C'est également la même solution en droit tunisien, les litiges relatives au transport maritime des passagers sont exclus du domaine de l'arbitrage, sauf si les parties concluent un compromise d'arbitrage, donc postérieurement a la naissance du litige : voir KAMEL BEN SALAH, “ An overview of the Tunisian Arbitration Regulations, JIA, 2000, n³, p.142.

${ }^{57}$ J. BEGUIN, G. BOURDEAUX, A. COURET, B. LE BASS, D. MAINGUY, M. MENJUCQ, H. RUIZ FABRI, C. SERAGLINI, J.M. SOREL, op. cit., p. 906.

58 N. NAJJAR, L'arbitrage dans les pays arabes face aux exigences du commerce international, LGDJ, 2004, p.174.

${ }^{59}$ V. P. LEVEL, Une première retouche au droit de l'arbitrage (loi du 5 juillet 1972), JCP, 1972.I.2494.

${ }^{60}$ V. TRUONG, " le droit applicable au fond dans les contrats internationaux de distribution : analyse des sentences arbitrales de la CCI, Bull. de la Cour international d'arbitrage de la CCI, vol. 12.n 1, $1^{\mathrm{er}}$ semestre 2001, p.40.

${ }^{61}$ Sentence CCI n8606 de 1997, RLAAI, ${ }^{\circ} 9$, p.20.

62 N. NAJJAR, L'arbitrage dans les pays arabes, op.cit., p.175.

${ }^{63}$ Sentence CCI n6500 de 1992, JDI, 1992.105, n. J.-J A ; RLAAI, nº 9, p.32 ; sentence CCI n 1250 de 1964, Rec. CCI, I, P.30.

${ }^{64}$ J. RUBELLIN-DEVICHI, L'arbitrage, nature juridique, droit interne et international privé, LGDI, Paris 1965, p. 60 ; P. LEVEL, L'arbitrabilité, précité, p.225 ; J.B. RACINE, L'arbitrage commercial international et l'ordre public, op. cit., p.48.
} 
judiciaire. Selon les termes de Savatier65, «quand la loi détermine une compétence judiciaire elle précise simplement la division du travail qu'elle établit entre les juges mandatés par elle. Elle n'envisage pas le cas où les plaideurs préfèrent se passer de ces juges ». Cette idée est confirmée par la jurisprudence française ${ }^{66}$.

En effet, l'arbitrage est délimité au litige en dehors de toute disposition juridictionnelle. Il n'est évidemment pas possible d'appliquer les mêmes règles aux tribunaux internationaux et aux tribunaux étatiques. Selon M. Racine ${ }^{67}$ "les règles relatives à l'arbitrabilité doivent rendre compte de l'originalité irréductible de l'arbitrage ». S'il devait prohiber automatiquement l'arbitrage dès lors qu'une matière incombe de manière impérative a un tribunal étatique, cela mènerait a limité considérablement son domaine, surtout dans les pays ${ }^{68}$, où la connaissance de bien des matières est, pour des considérations voire nationalistes, attribuées exclusivement aux juridictions étatiques ${ }^{69}$.

Il n'est pas sans intérêt de révéler à cet égard que la jurisprudence arbitrale internationale est pour sa part conforme à cette solution, dans une sentence partielle rendue en juin 1996 dans l'affaire $\mathrm{n} 8420$ concernant un contrat entre un mandant italien et un agent syrien, le droit applicable au fond étant le droit italien qui interdisait l'arbitrage. L'arbitre siégeant à Genève a déclaré le litige arbitrable en vertu de l'article 177 de la loi suisse sur le droit international privé. Les arbitres ont aussi remarqué que le règlement italien avait pour objet de protéger l'agent et que, dans l'affaire en question, c'était précisément ce dernier qui avait eu recours à la procédure arbitrale, l'exception d'incompétence ayant été soulevée par le mandant ${ }^{70}$.

Dans le même sens, un arbitre siégeant à Paris dans l'affaire CCI n ${ }^{\circ} 8195$, dans une sentence partielle rendue en septembre 1995 pour admettre l'arbitrabilité du litige, le tribunal a déclaré arbitrale un litige entre un agent libanais et un mandant français malgré l'invocation par le mandant du décret libanais n 34 du 5 août 196771 modifié par le décret n 9639 du 6 février 1975, et affirmé qu' : "il existe aujourd'hui un consensus international vis-à-vis des loi nationales des parties et sa soumission a la seule loi qui régit l'arbitrage (...) Plus précisément, il faut dire avant tout que le point de départ est le principe de l'autonomie de la clause d'arbitrage par rapport au contrat principal. Ce principe est généralement admis et ceci particulièrement en France, siège de l'arbitrage ».

L'arbitre a estimé que le principe d'autonomie de la convention d'arbitrage lui permettait de considérer comme valable une convention d'arbitrage ne violant ni les règles impératives du droit français ni les principes de l'ordre public international. Il a également souligné le fait que cette législation était destinée à protéger l'agent, alors qu'en l'occurrence c'est précisément ce dernier qui avait choisi de soumettre le différend a l'arbitrage ${ }^{72}$.

La solution est également suivie en matière de maritime commercial. Dans l'affaire CCI nº6194 de 1990 impliquant une partie jordanienne opposée a une partie coréenne, la partie jordanienne invoquait l'inaltérabilité du litige sur le fondement des dispositions de l'article 215 du Code maritime jordanien. Pour retenir sa compétence, le tribunal arbitral choisit, en premier lieu, pour la méthode de la propre Law et démontre qu'aucune loi en vertu des règles de conflit applicable, ne peut être considérer comme la propre Law applicable a la convention d'arbitrage.

Par conséquent, le tribunal arbitral admet la validité de la convention d'arbitrage insérée dans le contrat, en se fondant sur deux arguments essentiels : le premier est fondé sur le critère de l'inapplication de l'ordre public interne à l'arbitrage international: "les arbitres internationaux ne sont pas tenus de se soumettre aux règles d'ordre public interne d'un Etat déterminé». Plus convaincant, le second est fondé sur l'attente légitime des parties, ces dernières ayant conclu la convention à une date où la loi jordanienne sur le commerce maritime n'était pas encore en vigueur, la promulgation d'une loi postérieure à la date de conclusion du contrat ne peut porter atteinte au contrat conclu sous l'empire d'un droit antérieur plus libéral. Cette sentence présente le mérite d'illustrer la tendance actuelle de la jurisprudence arbitrale internationale, visant à exclure les règles restrictives a l'arbitrabilité an la matière ${ }^{73}$.

En conclusion, on peut constater que les critères admis dans certains Etats ${ }^{74}$, comme des règles de compétence judiciaire exclusive, sont insuffisants pour servir l'arbitrabilité des matières litigieuses du commerce international. Ces critères sont fortement en retrait tant par rapport aux besoins et pratiques commerciales que par rapport au droit comparé occidental.

\footnotetext{
${ }^{65}$ S. SAVANTIER, note sous Angers 27 mars 1953, D. 1945.407.

${ }^{66}$ CA de Colmar 21 septembre 1993, Ch. Morin SARL, M. Morin et autres, c. C.G. Morin : Rev. arb., 1994.348, n. D. Cohen ; Rev. jurisp.comm. 1994. 154, n.CH. JARROSSON.

${ }^{67}$ J.B. RACINE, L'arbitrage commercial international et l'ordre public, op. cit., p.48.

${ }^{68}$ Comme les pays arabes.

${ }^{69}$ N. NAJJAR, L'arbitrage dans les pays arabes, op.cit., P.176.

70 M.V. DER STOEL, The role of the OSCE high commissioner on national minorities in the field on conflict prevention, Martinus Nijhoff Publishers, 2003, p.196.

${ }^{71}$ Le décret-loi nº 34 du 5 Août 1967 modifié par le décret n 39 du 6 Février 1975 et par le décret-loi nº 73-83 du 9 Septembre 1983. V. site internet http://www.ccfranco-arabe.org/french/marche.php?code=113\&pays=12, le 27 mai 2009.

${ }^{72}$ F. BORTOLOTTI, Le contrat international d'agent commercial dans l'arbitrage de la CCI, Bulletin CCI, 2001, p.52.

73 J-L GOUTAL, La clause compromissoire dans les connaissements : la Cour de cassation française et la Cour suprême des Etats-Unis adoptent des solutions opposes, Rev, arb., 1996. 605.

${ }^{74}$ Comme par exemple les pays arabes : Jordanie, Syrie, Liban.
} 


\section{Les Références :}

[1] E. LOQUIN, Le contrôle du seul « caractère flagrant, effectif et concret » de la violation de l'ordre public international par la sentence, RTD Com. 2008.

[2] F. BORTOLOTTI, Le contrat international d'agent commercial dans l'arbitrage de la CCI, Bulletin CCI, 2001.

[3] F. NAMMOUR, Droit et pratique de l'arbitrage interne et international, 2e édition (refonte complète) LGDJ, BRUYLANT, DELTA, 2005.

[4] G. BONET ET CH. JARROSSON, L'arbitrabilité des litiges de propriété industrielle en droit français, in Arbitrage et propriété intellectuelle, Colloque de l'IRPI : Librairies techniques,

[5] G. HAZBOUN, For legal consultations, Middle East Newsletter, n¹. September. 2001.

[6] H. SINAY, La compétence exclusive, JCP. 1958 I 1451.

[7] J. RUBELLIN-DEVICHI, L'arbitrage, nature juridique, droit interne et international privé, LGDI, Paris 1965, p. 60 ; P. LEVEL, L’arbitrabilité, précité.

[8] J-B RACINE, L'arbitrage commercial international et l'ordre public, LGDJ, 1999.

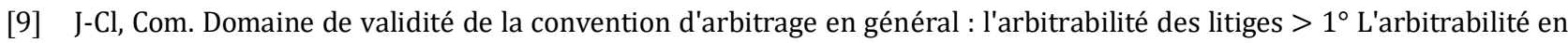
raison de la matière litigieuse. Cote 1-2009.

[10] J-Cl, Convention d'arbitrage, Domaine de validité de la convention d'arbitrage en général : l'arbitrabilité des litiges $>1^{\circ}$ L'arbitrabilité en raison de la matière litigieuse. Cote 1-2009.

[11] J-L GOUTAL, La clause compromissoire dans les connaissements : la Cour de cassation française et la Cour suprême des Etats-Unis adoptent des solutions opposes, Rev, arb., 1996.

[12] J-M. JACQUET ET PH. DELEBECQUE, Droit du commerce international, Dalloz, Cours, édition 3e, 2002.

[13] J-M. JACQUET et PH. DELEBECQUE, Droit du commerce international, Dalloz, Cours, édition 3e, 2002.

[14] L. Lévy, A. V. Schlaepfer, La suspension d'instance dans l'arbitrage international, Cah. arb., Gaz. Pal. №318.

[15] M. JOSSELIN-GALL, Arbitrage et propriétés intellectuelles, Droit et Patrimoine, $\mathrm{n}^{\circ} 105$, juin 2002.

[16] M.V. DER STOEL, The role of the OSCE high commissioner on national minorities in the field on conflict prevention, Martinus Nijhoff Publishers, 2003.

[17] Max Van Der Stoel, The role of the OSCE high commissioner on national minorities in the field on conflict prevention, Martinus Nijhoff Publishers, 2003.

[18] Max Van Der Stoel, The role of the OSCE high commissioner on national minorities in the field on conflict prevention, Martinus Nijhoff Publishers, 2003.

[19] N. NAJJAR, L'arbitrage dans les pays arabes face aux exigences du commerce international, L.G.D.J, 2004.

[20] N. NAJJAR, L'arbitrage dans les pays arabes face aux exigences du commerce international, L.G.D.J, 2004.

[21] N. NAJJAR, L'arbitrage dans les pays arabes face aux exigences du commerce international, LGDJ, 2004.

[22] P. LEVEL, Une première retouche au droit de l'arbitrage (loi du 5 juillet 1972), JCP, 1972.I.2494.

[23] P.Y. Tschanz et J. M. Vuillemin, « Chronique de jurisprudence étrangère. Suisse » : Rev. arb., 2002.

[24] S. SAVANTIER, note sous Angers 27 mars 1953, D. 1945.407.

[25] V. S. SALEH, commercial agency \& Distributorship in the Arab Middle East, op.cit., fasc. 4.

[26] V. TRUONG, « le droit applicable au fond dans le contrat internationaux de distribution : analyse des sentences arbitrales de la CCI, Bull. de la Cour international d'arbitrage de la CCI, vol. 12.n 1, 1er semestre 2001.

[27] W. ABDELGAWAD, L'arbitrage international et le nouveau règlement d'application des articles 81 et 82 CE : Rev. arb., 2004.

[28] Y. DERAINS, L'expérience de la Cour d'arbitrage de la Chambre de commerce internationale en matière de propriété industrielle, : Rev. arb., 1977. 